
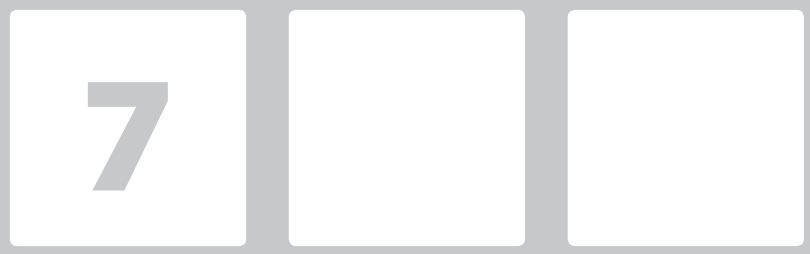

\title{
MARKETING PARA ORGANIZAÇÕES SEM FINS LUCRATIVOS DA CAUSA ANIMAL: ANÁLISE DA PREDISPOSI-ÇÃO À DOAÇÃO DE DINHEIRO E TEMPO
}

\section{Marketing for nonprofit organizations of animal cause: predisposition analysis to money and time donation}

\section{Anna Carolina Rodrigues Orsini}

Doutoranda no Programa de Pós-graduação em Administração da Universidade Federal da Paraíba. João Pessoa, PB. Brasil.

e-mail:carolorsini@hotmail.com

\section{Francisco José da Costa}

Doutor em Administração. Professor Associado Departamento de Administração da Universidade Federal da Paraíba. João Pessoa, PB. Brasil.e-mail:franzecosta@gmail.com

\section{RESUMO}

Este estudo objetivou analisar fatores individuais que levam à adoção de comportamentos de doação de dinheiro e tempo para a causa animal, considerando o papel de marketing no con-texto de ONGs. Foram lançadas nove hipóteses de pesquisa, testadas globalmente e por con-texto de coleta com residentes da cidade de João Pessoa (PB) por meio da modelagem linear de Theil-Sen. Entre os principais resultados, destaca-se que a percepção de colaboração com a causa como dever moral predispõe as pessoas a doar dinheiro e tempo. Além disso, as pessoas que percebem sua colaboração e a ação das ONGs como eficazes em promover o bem-estar de animais domésticos são mais propensas a adotar esses comportamentos. A pesquisa contribui para a prática de gestão de marketing em organizações sociais voltadas à causa animal, ao pas-so que incentiva a discussão de marketing no interesse social e de causas sociais mais específi-cas em âmbito acadêmico.

Palavras-chave: Causa animal. Doação. Marketing. ONGs.

\section{ABSTRACT}

This study aimed to analyze individual factors that lead to the adoption of behaviors for do-nating money and time to the animal cause, considering the role of marketing in the context of NGOs. Nine hypotheses were launched, tested globally and by context of collection with res-idents of João Pessoa (PB) through the linear modeling of Theil-Sen. Among the main results, it is emphasized that the perception of collaboration with the cause as a moral duty predispos-es people to donate money and time. In addition, people who perceive their collaboration and NGO action as effective in promoting the welfare of domestic animals are more likely to adopt such behaviors. The research contributes to the practice of marketing management in social organizations focused on the animal cause, while encouraging the discussion of market-ing in the social interest and of more specific social causes in academic scope.

Keywords: Animal cause. Donation. Marketing. NGOs. 


\section{INTRODUÇÃO}

A proposta de ampliar a compreensão de marketing para abarcar questões além do âmbito empresarial (KOTLER; LEVY, 1969) trouxe discussões e implicações importantes para a gestão das organizações sociais. Desde então, a academia tem se esforçado para contribuir com a gestão dessas organizações, investigando principalmente comportamentos de interesse, como por exemplo, a doação (BASIL; RIDGWAY; BASIL, 2008; BEKKERS, 2010; BRYANT et al, 2003; LEE;CHANG, 2007; KOTTASZ, 2004; NEUMANN, 2010; SLYKE; BROOKS, 2005; WIEPKING; MAAS, 2009).

Entretanto, a maioria dos esforços empreendidos é genérica, no sentido de não considerar as especificidades das diversas causas existentes e o contexto de atuação para essas entidades. Ademais, apesar do terceiro setor reconhecer a importância da atividade de marketing, não busca ou não consegue incorporá-la efetivamente em sua gestão (POPE; ISELY; ASAMOA-TUTU, 2009), mesmo lidando com um ambiente equiparável ao empresarial, com grande crescimento e diversificação no número de entidades atuantes (em 2016 a estimativa era de 303 mil ONGs existente no Brasil, mais de 60 mil na região Nordeste) e maior dificuldade de angariar recursos (BLERY; KATSELI; TSARA, 2010; INSTITUTO DE PESQUISA ECONÔMICA APLICADA (IPEA), 2014; NEUMANN, 2010; SLYKE; BROOKS, 2005).

Para causas como a de proteção ambiental e de defesa dos animais é ainda mais desafiador encontrar na literatura direcionamentos relacionados à sua gestão de marketing especificamente. Isto ocorre embora essas organizações tenham apresentado crescimento de $14,7 \%$ acima da média nacional até 2010 , como mostrou um levantamento feito pelo Instituto Brasileiro de Geografia e Estatística (IBGE) (2012), em parceria com o IPEA, Associação Brasileira de Organizações Não Governamentais (ABONG) e o Grupo de Instituições Fundações e Empresas (GIFE). Esse crescimento vem, possivelmente, como resposta a questões relacionadas à causa animal e pode estar associado ao fato de o Brasil ser a quarta nação em população total de animais de estimação, segunda maior em população de cães e gatos, e possuir cerca de 30 milhões de animais abandoados (AGÊNCIA
DE NOTÍCIAS DE DIREITOS ANIMAIS (ANDA), 2014; ASSOCIAÇÃO BRASILEIRA DA INDÚSTRIA DE PRODUTOS PARA ANIMAIS DE ESTIMAÇÃO (ABINPET), 2013).

A partir do exposto, e visando trazer uma contribuição para a gestão dessas organizações em uma perspectiva de marketing, nesse artigo tomou-se por desafio explorar as razões que levam as pessoas a adotar comportamentos favoráveis à causa animal. Em particular, os comportamentos de doação de dinheiro e tempo (trabalho voluntário). Esse recorte buscou atender às recomendações de pesquisas de Pope, Isely e Azamoa-Tutu (2009), Neumann (2010) e Bekkers e Wiepking (2011), no sentido de estudar causas de maneira mais específicas, oportunizando a discussão acadêmica dessa temática ainda pouco explorada. Essa pesquisa contribui ainda para reforçar a perspectiva mais profissional da atuação do terceiro setor, pensando na oferta de produtos sociais e na compreensão de comportamentos de consumo dessa natureza, ou seja, em uma perspectiva de marketing ampliada. Sendo assim, tem uma proposta original e relevante por contribuir com o debate de gestão de marketing em um contexto de causa social, tratando especificamente da causa animal, tema que repercute cada vez mais em cenário nacional.

A seguir temos o embasamento teórico do estudo, seguido do detalhamento acerca dos procedimentos metodológicos adotados. Posteriormente, discutimos os resultados alcançados e as considerações finais sobre o trabalho, enfatizando limitações e recomendações para pesquisas futuras.

\section{REFERENCIAL TEÓRICO}

Nesse item apresentam-se, algumas considerações sobre marketing de organizações do terceiro setor, e, em seguida, realiza-se a fundamentação das hipóteses testadas no estudo empírico.

\subsection{Marketing para organizações do terceiro setor}

A disciplina de marketing, principalmente a partir dos anos 1980, passou a envolver novas discussões com a inclusão de temáticas de interesse social 
(BURGUETE, 2004). À medida que o ambiente se tornou mais competitivo para as organizações sem fins lucrativos, evidenciou-se a necessidade de uma gestão mais profissionalizada com apoio imprescindível de atividades de marketing. Entendemos então que marketing para organizações sem fins lucrativos é uma orientação de gestão que ajuda essas organizações a tornar suas atividades mais eficientes por promover uma perspectiva com foco também em elementos externos e, assim, direcionar esforço para gerenciamento de imagem, promoção da causa e dos benefícios sociais, atração e retenção de recursos, dentre outros (WYMER; KNOWLES; GOMES, 2006).

De fato, é essencial apropriar-se do conhecimento de marketing para promover trocas mais efetivas e desenvolver atividades de maneira mais eficiente e eficaz, observando a compatibilidade entre o que se oferta, a demanda do público-alvo e os benefícios provenientes da atuação organizacional, a fim de estimular comportamentos convergentes aos objetivos propostos (DRUCKER, 2006). Em termos práticos, para Andreasen e Kotler (2008), o processo de planejamento estratégico de marketing em organizações sem fins lucrativos envolve três grandes etapas: análise, estratégia e implementação. A análise abarca questões relativas ao ambiente organizacional (missão, objetivos, cultura, forças e fraquezas) e ao ambiente externo (público-alvo, competitividade, oportunidades e ameaças). Essa primeira etapa serve de base para estruturação do conjunto principal de estratégias de marketing (mix de marketing, segmentação e posicionamento) que são implementadas segundo determinados parâmetros de desempenho para avaliação.

No que se refere ao público-alvo, é preciso compreender os diversos tipos de público com que lidam, já que as trocas realizadas alcançam não apenas ofertantes e demandantes, mas também outros públicos indiretos como os animais, o poder público e a sociedade. Os públicos provedores (apoiadores, fornecedores, reguladores) são aqueles que direcionam e fomentam as ações organizacionais. Já os públicos internos são aqueles que desenvolvem as ações planejadas, enquanto os públicos agentes (distribuidores) disseminam as ações para que alcance os públicos consumidores (clientes e público em geral) (ANDREASEN; KOTLER, 2008; KOTLER,
1994). Dolnicar e Lazarevski (2009) argumentam que a orientação das práticas e atividades dessas organizações precisa considerar a satisfação dos clientes, que nesse caso, conforme mencionado, se configuram em mais de um público. No contexto da causa animal, os doadores e voluntários assumem diversos papéis, de modo que é preciso que a organização desempenhe um esforço de marketing estratégico e efetivo para desenvolver ações e promover a causa, conquistando e mantendo esses agentes de interesse.

A análise de mercado, considerando seus limites, segmentos e posicionamento, também é uma atividade de marketing importante para as organizações sem fins lucrativos que complementa a identificação dos públicos de interesse. A segmentação permite a identificação dos perfis de interessados e potenciais colaboradores da causa em questão e, consequentemente, oportuniza uma maior adequação, através do posicionamento, entre o produto, em seu sentido mais amplo, e o mercado, arena em potencial para a troca em seu sentido mais abrangente (ANDREASEN; KOTLER, 2008; KOTLER, 1994).

O mix de marketing (4Ps), por sua vez, é dimensionado de forma diferenciada. O produto abrange bens, serviços, lugares, ideias, pessoas, organizações e os custos e benefícios gerais envolvidos. Quanto ao preço, é preciso considerar os custos econômicos associados a sacrifícios de crenças, valores e hábitos, antigos padrões de comportamento e de tempo e energia. No que tange à distribuição, assim como as empresas, as organizações que não visam lucro devem adotar estratégias para garantir acesso ao seu produto. Por fim, o aspecto promocional, que envolve todo o arranjo de comunicação integrada de marketing, deve ser direcionado a fim de estimular a ação de comportamentos favoráveis à causa, conquistar e manter parceiros, doadores e voluntários e angariar recursos (ANDREASEN; KOTLER, 2008; KOTLER, 1994).

A partir de uma perspectiva estratégica de comunicação de marketing, a ação de captação de recursos (fundraising) deve ser percebida não como um pedido baseado em motivos filantrópicos, mas como uma troca de valor compatível com a necessidade do doador. Čačija (2013) fundamenta que as organizações sem fins lucrativos precisam adotar uma perspectiva de captação de recursos pautada na visão de marketing estratégico, buscando gerar maior 
confiança, compromisso e relações de longo prazo com suas fontes de financiamento a fim de tornar sua existência mais sustentável. Um dos grandes desafios é trazer indicadores objetivos sobre os resultados alcançados, dada a natureza intangível das atividades que constituem esse setor social, como essência de qualquer serviços prestados.

Pesquisas mais recentes buscaram compreender questões relativas a marketing e/ou direcionar a gestão das organizações sociais envolvidas com a causa animal, com um foco mais preciso no aspecto promocional. O estudo de caso de Blery, Katseli e Tsara (2010) buscou examinar a prática de marketing na ONG WFF- Grécia, utilizando como parâmetro a composição do seu mix de marketing. Os estudos de Haynes, Thornton, Jones (2004), Zucco, Krauss e Fernandes (2013) e Orsini, Barboza e Costa (2015) pesquisaram a relação entre apelos publicitários e adoção de comportamentos de colaboração com a causa. Sendo assim, se faz oportuno aprofundar as discussões acerca dos fatores que potencializam os comportamentos de doação de recursos, como dinheiro e tempo, a fim de estimular uma discussão de gestão de marketing mais sólida para organizações dessa natureza.

\subsection{Fatores antecedentes aos comportamentos de doação}

Nessa pesquisa, tem-se como pressuposto que os principais comportamentos de interesse para as organizações sem fins lucrativos voltadas à causa animal são: a doação e a adoção. Todavia, o foco desse estudo é no comportamento de doação, uma categoria relevante para que as organizações do terceiro setor consigam alcançar seus objetivos e missão (ČAČIJA, 2013). O ato de colaborar envolve desde a compreensão da situação de necessidade até a ação efetiva de ajuda e é influenciada por diversos fatores que a inibem ou a estimulam (GUY; PATTON 1989). A doação, como um tipo de colaboração, é um comportamento pró-social relacionado à promoção de ajuda a beneficiários necessitados. Logo, possui certo grau de complexidade em seu entendimento, por envolver fatores de natureza individual, situacional e organizacional (BRANCO, 1983; WIEPKING;
MAAS, 2009). Souza e Medeiros (2012) salientam que a doação de tempo, caracterizada pelo trabalho voluntário, é ainda mais diversificada no que tange às suas motivações.

Em geral, os estudos têm focado principalmente em analisar aspectos sociodemográficos como fatores de explicação para o comportamento de doação (BASIL; RIDGWAY; BASIL, 2008; BEKKERS, 2010; BRYANT et al., 2003; KOTTASZ, 2004; NEUMANN, 2010). Mas, já temos algumas evidências na literatura, ainda que em menor proporção, da exploração de aspectos individuais intrínsecos como motivadores para esse comportamento. Demonstram-se esses aspectos a seguir com a enunciação de algumas hipóteses para fundamentação do estudo empírico.

Como primeiro aspecto, pesquisas apontam que experiências vivenciadas com animais ao longo da vida influenciam a atitude e o comportamento das pessoas em relação aos animais (ZAHN-WAXLER; HOLLENBECK; RADKE-YARROW, 1985). Bryant et al. (2003) sugerem que pessoas com maior capital humano, isto é com maior acúmulo de experiências, habilidades e educação com orientação filantrópica ou pró-social, são mais propensas a doar, e Blouin (2012) compreende que o papel e o significado do pet para o indivíduo estão associados à construção social sobre o animal, como também ao grau de exposição a animais e às experiências vivenciadas com eles, principalmente na infância. A partir dessas sinalizações, enuncia-se a seguinte hipótese:

\section{H1 - A avaliação das experiências prévias com animais influencia positivamente 0 comportamento de doação.}

Em paralelo, há indicações de que o grau de socialização, a consideração das expectativas sociais e o desejo de agir em conformidade social, além da convivência e exemplos de grupos sociais de referência, como família, predispõem as pessoas a comportamentos pró-sociais, como é o caso da doação (BRANCO, 1983; BRYANT et al., 2003; GUY; PATTON, 1989; MARTIN; RANDAL, 2008; WIEPKING; MAAS, 2009). Com isso, definimos uma segunda hipótese de pesquisa, a saber: 


\section{H2 - A interação com grupos sociais envolvidos com a causa animal influencia positivamente o comportamento de doação}

Cheung e Chan (2000) e Skyle e Brooks (2005) verificaram que a percepção de autoeficácia e efetividade, intimamente relacionada às ideias de Guy e Patton (1989) sobre a percepção de habilidade para ajudar predispõem as pessoas à colaboração social com doação. Os estudos de Bryant et al. (2003), Lee e Chang (2007), Wiepking e Maas (2009), Bekkers (2010) e Bekkers e Wiepking (2011) também corroboram com essa indicação. Sendo assim, a percepção de eficácia envolve a confiança na própria capacidade de exercer o comportamento de colaboração e no alcance efetivo de resultados a partir dele. Posto isso, indica-se a seguinte hipótese:

\section{H3 - A percepção de eficácia para a causa animal influencia positivamente 0 comportamento de doação}

Os estudos de Lee e Chang (2007), Basil, Ridgway e Basil (2008), Wiepking e Maas (2009) e Bekkers (2010) enfatizam a importância da empatia nos comportamentos de ajuda e doação, indicando que pessoas altruístas são mais propensas a ajudar, já que reagem emocionalmente e buscam compreender a condição do outro. O conceito de empatia por animais, de acordo com os estudos de Basil, Ridgway e Basil (2008), Lee e Chang (2007) e Rothgerber e Mican (2014), reflete a sensibilidade em relação aos animais quando buscamos compreender suas emoções e reações em determinadas circunstâncias, nos colocando em seu lugar. Isso nos leva à enunciação da quarta hipótese da pesquisa:

\section{H4 - A empatia por animais influencia positivamente o comportamento de doação}

A literatura também indica a relação entre o senso de obrigação e dever moral e comportamentos de doação (CHEUNG; CHAN, 2000; LEE; CHANG, 2007; SKYLE; BROOKS, 2005), e a ideia de consideração moral dos animais na sociedade (REGAN, 1983; 1997; SINGER, 2010). Souza e Medeiros (2012) indicam a existência de uma categoria de motivações para trabalho voluntário voltada para a preocupação com a sensação de dever cumprido e responsabilidade cívica e moral. A partir disso, considera-se pertinente enunciar a hipótese a seguir:

\section{H5 - O senso de obrigação moral em relação aos animais influencia positivamente 0 comportamento de doação}

Também está bem estabelecido que o fenômeno de antropomorfização, isto é, atribuição de características, motivações e emoções tipicamente humanas a agentes não humanos, é um fator indicativo da percepção de semelhança entre pessoas e animais (BLOUIN, 2012; EPLEY; WAYTZ; CACIOPPO, 2007; HOLBROOK, 2008), e há indicações na literatura de que a percepção de semelhança entre doador e beneficiário aumentam as chances de doação (BLOUIN, 2012; BRANCO, 1983; HOLBROOK, 2008). Nesse sentido, pode-se enunciar a sexta hipótese da pesquisa:

\section{H6 - A percepção de semelhança com animais influencia positivamente o comportamento de doação}

Singer (2010) argumenta que os animais são seres sencientes, ou seja, seres dotados de sensibilidade capazes de sentir dor/sofrimento e prazer/bem-estar. Haynes, Thornton e Jones (2004), Zucco, Krauss e Fernandes (2013) e Orsini, Barboza e Costa (2015) discutem que o sofrimento dos animais é um tipo de apelo com potencial de mobilizar comportamentos de colaboração. A partir disso, definiu-se a seguinte hipótese:

\section{H7 - O reconhecimento da capacidade de sofrimento animal influencia positivamente $o$ comportamento de doação}

Ademais, a Portaria no 93 do Instituto Brasileiro do Meio Ambiente e dos Recursos Naturais Renováveis (1998) declara explicitamente que a fauna doméstica possui características em estreita dependência do homem, ideia ligada diretamente à conferência de deveres do homem para com eles (MEDEIROS; GRAU NETO, 2012; 
SINGER, 2014) e à discussão sobre a proteção e direito dos animais e sua eficácia para estimular comportamentos de colaboração (BRASIL, 1998; ORSINI; BARBOZA; COSTA, 2015; UNESCO, 1978). Considerando o exposto, enunciam-se as duas últimas hipóteses formuladas:

\section{H8 - O reconhecimento de dependência animal influencia positivamente $o$ comportamento de doação}

\section{H9 - O reconhecimento do direito animal influencia positivamente o comportamento de doação}

Apresentadas as hipóteses do estudo, expomos os encaminhamentos metodológicos adotados na pesquisa.

\section{PROCEDIMENTOS METODOLÓGICOS}

Para o teste empírico das hipóteses, optou-se por mensurar os construtos a partir da tradução e adaptação de escalas existentes. Alguns itens, no entanto, foram elaborados a partir da apropriação de conteúdo da literatura e experiências anteriores de pesquisa com a temática, por não haver escalas validadas em estudos anteriores que se adequassem aos construtos estudados. Para esses itens, realizou-se o processo de validação de face e conteúdo com quatro pesquisadores da área marketing para ratificar a pertinência e representatividade desses, conforme sugerido por Costa (2011). No intuito de consolidar o instrumento, também foi feito um teste preliminar com 10 respondentes a fim de verificar a necessidade de ajustes quanto à forma e compreensão dos itens. Após essa etapa, coletaram-se os dados por meio de questionário composto por questões de descrição da amostra e de mensuração dos construtos definidos.

Decidiu-se por mensurar dois construtos (avaliação das experiências prévias com animais e interação com grupos sociais envolvidos com a causa animal) em escala com single item, tendo em vista a consistência da estratégia para verificação de construtos e a busca por evitar correlações excessivas (redundâncias) e desnecessárias. Os demais construtos apresentaram pelo menos dois itens, conforme a recomendação predominante em pesquisa (COSTA, 2011). Foram utilizadas escalas phrase completion e de concordância (de 11 pontos), considerando o alinhamento necessário entre medições e construtos estudados.

O construto de avaliação das experiências prévias com animais foi mensurado pelo único item 'Ao longo da vida, minhas experiências com pets (animais domésticos) foram preponderantemente..., elaborado com base em Blouin (2012) e Zahn-Waxler, Hollenbeck e Radke-Yarrow (1985). A interação com grupos sociais envolvidos com a causa animal também foi mensurado por single item: 'A minha convivência com pessoas que atuam pela causa animal é..., baseado no modelo de escala de Rossiter (2009). Para a percepção de eficácia para a causa animal, utilizou-se os itens 'Eu acredito que tenho condições de contribuir (com dinheiro, ou trabalho, ou adoção etc.) para a causa animal em um nível...' e 'Em minha leitura, o envolvimento das pessoas com a causa animal (doando dinheiro, tempo etc.) pode gerar um efeito..., desenvolvidos com base nas escalas de Basil, Ridgway e Basil (2008) e Cheung e Chan (2000).

Os itens relacionados à empatia por animais foram 'Minha capacidade de compreender as emoções sentidas pelos animais é...' e 'A minha sensibilidade em entender as reações e me colocar no lugar dos animais é..., formulados a partir dos estudos de Basil, Ridgway e Basil (2008), Lee e Chang (2007) e Rothgerber e Mican (2014). A mensuração do construto de senso de obrigação moral em relação aos animais, por sua vez, se deu por meio dos itens 'Para mim, contribuir com a causa animal está de acordo com meus princípios morais em um nível...' e 'Meu sentimento de obrigação moral em contribuir com a causa animal é..., elaborados com base em Beldad, Snip e Van Hoof (2014) e Cheung e Chan (2000). Os estudos de Epley,Waytz e Cacioppo (2007) e Rothgerber e Mican (2014) fundamentaram a elaboração dos itens 'Os animais domésticos são como entes queridos (familiares, amigos etc.)' e 'Pessoas e animais domésticos são bastante semelhantes (características, emoções e comportamentos)' relacionados à percepção de semelhança com animais. 
Para o reconhecimento da capacidade de sofrimento animal foram utilizados, segundo o aporte teórico de Regan $(1983,1997)$ e Singer $(2010)$, os itens 'Os animais abandonados vivenciam sofrimento físico' e 'Os animais abandonados vivenciam sofrimento psicológico'. Para mensuração do reconhecimento da dependência animal utilizou-se os itens 'Os animais domésticos dependem das pessoas para sobreviverem' e 'Os animais domésticos dependem das pessoas para terem bem-estar' a partir das considerações de IBAMA (1998), Medeiros e Grau Neto (2012) e Singer (2014). Por fim, os itens 'Os animais têm direito a uma vida digna sem exploração, maus-tratos e abandono' e 'As pessoas precisam reconhecer e apoiar os direitos dos animais a terem uma vida sem exploração, maus-tratos e abandono', desenvolvidos a partir de Lei no 9.605 (1998), Medeiros e Grau Neto (2012), Orsini, Barboza e Costa (2015) e Regan $(1983,1997)$, mensuraram o reconhecimento do direito animal. Os itens das variáveis preditas estão especificados na sessão de análise e foram baseados no estudo de Basil, Ridgway e Basil (2008).

O critério de seleção de amostra foi definido pela maioridade dos respondentes (18 anos), condição que indica independência e capacidade de adotar comportamentos dessa natureza. Além disso, foram selecionados apenas residentes da cidade de João Pessoa (PB), haja vista a necessidade de se considerar o contexto de estudo e suas particularidades. A amostra contemplou um total de 389 questionários, seguindo o padrão observado nos estudos quantitativos relacionados ao tema. Seguindo o modelo internacional e considerando as limitações convencionais da pesquisa acadêmica, para definir o tamanho da amostra não probabilística observou-se, além da quantidade média de respondentes de estudos anteriores com essa abordagem, a adequação desse quantitativo em relação ao uso consistente das técnicas de análise (HAIR JR et al., 2014). Foram aplicados questionários online, via redes sociais, e presenciais, no intuito de tornar o processo de coleta mais simplificado sem comprometer os resultados, já que o objetivo primordial do estudo é testar as relações entre os fatores antecedentes e os comportamentos pró-animal definidos.

A coleta online contemplou a inserção do questionário através da ferramenta Google Docs com publicação e compartilhamento na rede social virtual Facebook e totalizou 228 respostas. Presencialmente, foram aplicados 161 questionários em diversos locais públicos com grande movimentação na cidade de João Pessoa, buscando uma heterogeneidade que se aproxime da heterogeneidade que se observa no universo real de respondentes. Após a coleta, os dados foram lançados no software SPSS para a realização das análises preliminares de limpeza dos dados, através da verificação de valores perdidos e outliers, o que resultou no total de 367 respostas válidas para os testes. No geral, a amostra obteve maior representatividade de respondentes jovens e jovens adultos (quase $85 \%$ dos respondentes com idade entre 18 e 41 anos), com alto nível de escolaridade (54,2\% com ensino superior e 34,3\% com pós-graduação) e que já teve ou tinha, no momento da realização da pesquisa, algum animal doméstico. Também houve predominância de respondentes do sexo feminino (65,7\%). Esse perfil era esperado, considerando que há uma indicação de maior envolvimento de jovens mulheres com a causa animal (NEUMANN, 2010).

A análise dos dados possui dois momentos centrais. O primeiro envolve as análises preliminares de agregação e descrição dos itens relacionados às variáveis preditas, como também testes para verificar se havia diferença estatisticamente significativa nas variáveis-resposta entre os contextos de coleta, dado que há um pressuposto de diferenciação por espontaneidade na coleta. Considerando a constatação da diferença, no segundo momento testaram-se as hipóteses elaboradas por meio da modelagem linear robusta de Theil-Sen, Para operacionalizar essas etapas foram utilizados os softwares SPSS e R. Esse encaminhamento buscou atender ao critério de adequação entre o objeto de estudo e forma de análise (HAIR JR et al., 2014). A seguir, discorre-se sobre os resultados obtidos.

\section{ANÁLISE DOS RESULTADOS}

Esse tópico, conforme exposto previamente, contempla a análise preliminar e descritiva dos construtos e a análise das relações previstas testadas por modelagem linear robusta de Theil-Sen. 


\subsection{Análise preliminar e descritiva}

A primeira etapa de análise envolveu a verificação de adequação e consistência na mensuração das variáveis dependentes, já que as variáveis independentes não contemplaram pressuposição anterior de que tinham condições refletivas em relação aos construtos de doação ou foram medidas por single item. No primeiro caso, as variáveis independentes foram agregadas diretamente. Conforme exposto na Tabela 1 , as extrações sinalizaram boa adequação dos itens para efeito de mensuração dos dois construtos (doação de dinheiro e doação de tempo para a causa animal) e boa estrutura psicométrica.

Tabela 1 Escores para agregação de itens

\begin{tabular}{|c|c|c|c|}
\hline Construto & Itens & Esc. Fatorial & Alpha \\
\hline \multirow{3}{*}{$\begin{array}{c}\text { Doação de dinheiro } \\
\text { KMO=0,741 } \\
\text { Teste de Bartlett }= \\
\text { p-valor }<0,001 \\
\left(\text { com } x^{2}=854,226 \text { e } 3 \text { gl) }\right.\end{array}$} & $\begin{array}{l}\text { A probabilidade de eu fazer uma doação de dinheiro para a causa } \\
\text { animal é de nível }\end{array}$ & 0,928 & \multirow{3}{*}{0,923} \\
\hline & $\begin{array}{l}\text { A probabilidade de eu contribuir com dinheiro para a causa animal é } \\
\text { de nível }\end{array}$ & 0,914 & \\
\hline & Meu desejo de doar dinheiro para a causa animal é de nível & 0,952 & \\
\hline \multirow{3}{*}{$\begin{array}{c}\text { Doação de tempo } \\
\text { KMO = 0,772 } \\
\text { Teste de Bartlett }= \\
\text { p-valor }<0,001 \\
\left(x^{2}=1008,119 \text { e } 3 \text { gl }\right)\end{array}$} & A chance de fazer trabalho voluntário em favor da causa animal é de nível & 0,953 & \multirow{3}{*}{0,944} \\
\hline & $\begin{array}{l}\text { A chance de eu me envolver em algum trabalho a favor da causa animal } \\
\text { é de nível }\end{array}$ & 0,946 & \\
\hline & $\begin{array}{l}\text { Minha vontade de fazer alguma ação (trabalho) voluntária em favor dos } \\
\text { animais é de nível }\end{array}$ & 0,946 & \\
\hline
\end{tabular}

Fonte: Dados da pesquisa (2015).

A análise dos construtos apresentou a emergência de um único fator com autovalor maior que 1 e explicação da variância total considerada muito satisfatória (86,79\% e 89,9\% respectivamente), assim como os escores fatoriais (todos acima de 0,9). Além disso, o valor dos coeficientes alpha de Cronbach extraídos sinalizam alta confiabilidade na escala.

Desse modo, procedemos à agregação dos itens a partir da média dos escores por respondente. Nas variáveis agregadas, conforme o Apêndice A, extraímos como medidas de posição a média simples, e as médias aparada e winsorizada, ambas em 10\%, além dos três quartis; para avaliar a dispersão e formato dos dados extraímos, respectivamente, a medida de desvio padrão e assimetria e curtose de Pearson. Obtivemos medidas de posição central moderadas, com dispersão elevada entre as respostas e medidas de formato que indicando leve assimetria à esquerda e curtose pouco convergente com o parâmetro de normalidade (considerando o parâmetro de extração do R, em que valores de normalidade estão centrados em 3).

Os resultados para a análise descritiva dos construtos preditores, também apresentados no
Apêndice A, sinalizam que, em geral, há elevado nível de experiências prévias com animais, de empatia e de percepção de semelhanças com os mesmos, como também para o reconhecimento de sofrimento, de dependência e de direitos; houve um nível moderado de percepção de eficácia, de senso de obrigação moral e de interação com grupos sociais envolvidos com a causa. Os níveis de dispersão das medidas foram baixos, e na maioria dos construtos não foi possível assegurar normalidade na distribuição dos dados (segundo os parâmetros centrais 0 e 3 de assimetria e curtose do R).

Seguindo nessa primeira etapa de análise, verificou-se a possibilidade de diferença estatística de comportamento nas variáveis respostas de acordo com o contexto de coleta (online ou presencial). Para tal, realizaram-se os testes de Kolmogorov-Smirnov e Cramer-von Mises (em ambos os testes a hipótese nula é de que as distribuições são idênticas). Assim, para as variáveis de predisposição em doar dinheiro e doar tempo para a causa animal a hipótese nula foi rejeitada no teste de Kolmogorov-Smirnov ( $\mathrm{D}=0,153$, $\mathrm{p}$-valor $=0,032 ; \mathrm{D}=0,219, \mathrm{p}$-valor $=0,000)$ e no teste 
de Cramer-von Mises $(\mathrm{T}=0,888$, $\mathrm{p}$-valor $=0,007$; $\mathrm{T}=1,640$, p-valor $=0,000)$, indicando a existência de diferença de distribuição.

Para compreender como essa diferenciação estava configurada, operacionalizaram-se os testes de comparação de medidas de posição (média e mediana). A partir disso, constatou-se que há diferença em média e em mediana ( $\mathrm{p}$-valor $<0,01$ ) das variáveis de doação de dinheiro e tempo entre os contextos de coleta, com medidas maiores no contexto online, sinalizando que a predisposição dos respondentes online é maior em colaborar com a causa. Possivelmente, isso reflete a característica de obtenção de respostas espontâneas no processo de coleta de dados online. Com essa constatação, realizou-se a análise descritiva e os testes de hipóteses tanto globalmente, contemplando a amostra em sua totalidade, como por contexto de coleta.

A partir dessa análise, é possível afirmar que no geral há uma postura positiva e favorável em relação aos animais e que há nível moderado de predisposição em adotar comportamentos favoráveis à causa animal. A seguir, apresentam-se os testes das hipóteses formuladas para compreender a possível relação entre esses fatores.

\subsection{Teste de hipóteses}

Para analisar as hipóteses formuladas, utilizou-se primeiramente a técnica de modelagem de regressão normal linear múltipla. Apesar da constatação de boa consistência e explicação geral do modelo, os pressupostos não foram plenamente atendidos e, por esta razão, aplicou-se a modelagem de regressão linear de Theil-Sen. Essa modelagem é um método computacionalmente intensivo, e consiste em extrair, por bootstrapping, um grande número de subamostras da amostra original, cada uma com o tamanho mínimo necessário para extração de estimadores dos parâmetros de regressão por mínimos quadrados; em seguida, são extraídas medidas para os parâmetros com base nas medianas dos parâmetros estimados em cada extração. O uso de boostrapping permite a flexibilização de potenciais pressupostos (como aqueles da modelagem normal linear) e a definição de parâmetros com base nas medianas torna a técnica robusta a problemas de outliers (tanto na forma de pontos de alavanca quanto de influência) (cf. WILCOX, 2012). A seguir, detalham-se os resultados obtidos.

\subsubsection{Predisposição em doar dinheiro para a causa animal}

Por meio da modelagem de Theil-Sen, conforme sinalizam os estimadores globais apresentados na Tabela 2, confirmou-se a influência dos fatores 'senso de obrigação moral em relação aos animais' ( $\beta=0,583$, $\mathrm{p}<0,001)$ e 'percepção de eficácia para a causa animal' $(\beta=0,461, \mathrm{p}<0,001)$ para explicar a doação de dinheiro para causa animal. $O$ 'reconhecimento do direito animal' $(\beta=0,350, p=0,057)$ emergiu como fator de influência positiva marginal. A medida de ajuste do modelo (Pseudo- $\mathrm{R}^{2}$ ) indicou nível de ajuste global razoável $(0,445)$.

No contexto online, os mesmos construtos foram confirmados, porém com um ajuste menor (Pseudo- $\mathrm{R}^{2}=0,392$ ). Ainda assim, houve influência marginal mais significativa do construto de 'reconhecimento do direito animal' ( $\beta=0,523, \mathrm{p}<0,05)$, o que não ocorreu no contexto presencial, em que foram mantidos apenas os construtos de senso de obrigação moral $(\beta=0,528, \mathrm{p}<0,01)$ e percepção de eficácia $(\beta=0,599, \mathrm{p}<0,001)$ com nível de explicação similar à estimação global (Pseudo- $\left.\mathrm{R}^{2}=0,455\right)$.

\subsubsection{Predisposição em doar \\ tempo para a causa animal}

De acordo com a Tabela 3, confirmou-se a influência dos fatores de senso de obrigação moral $(\beta=0,720, p<0,001)$, percepção de eficácia $(\beta=0,193$, $\mathrm{p}<0,01)$, reconhecimento do direito animal $(\beta=0,439, p=0,01)$, interação com grupos sociais envolvidos $(\beta=0,216, p<0,01)$ e influência marginal de experiências prévias $(\beta=0,100, \mathrm{p}<0,05)$ sobre a predisposição em doar tempo para a causa animal. A medida de ajuste do modelo (Pseudo- $\mathrm{R}^{2}$ ) também indicou bom nível de explicação $(0,605)$. 
Tabela 2 Estimadores do modelo Theil-Sen para doação de dinheiro

\begin{tabular}{|c|c|c|c|c|c|}
\hline \multicolumn{6}{|c|}{ Estimadores do Modelo Global (Pseudo- $\mathbf{R}^{2}=0,445$ ) } \\
\hline Preditor & LI-IC & LS-IC & Estimador & Erro padrão & p-valor \\
\hline Intercepto & $-6,949$ & $-1,725$ & $-4,210$ & 0,013 & 0,000 \\
\hline Experiências prévias & $-0,086$ & 0,163 & 0,029 & 0,062 & 0,554 \\
\hline Convivência com engajados & $-0,064$ & 0,297 & 0,120 & 0,093 & 0,244 \\
\hline Percepção de eficácia & 0,275 & 0,671 & 0,461 & 0,100 & 0,000 \\
\hline Empatia pelos animais & $-0,192$ & 0,180 & $-0,057$ & 0,095 & 0,681 \\
\hline Senso de obrigação moral & 0,331 & 0,748 & 0,583 & 0,104 & 0,000 \\
\hline Percepção de semelhança & $-0,101$ & 0,221 & 0,070 & 0,084 & 0,451 \\
\hline Rec. da capacidade de sofrimento & $-0,358$ & 0,094 & $-0,136$ & 0,114 & 0,280 \\
\hline Rec. da dependência & $-0,162$ & 0,217 & 0,017 & 0,099 & 0,811 \\
\hline Reconhecimento do direito & $-0,008$ & 0,741 & 0,350 & 0,196 & 0,057 \\
\hline \multicolumn{6}{|c|}{ Estimadores dos Dados da Coleta Online (Pseudo- $\left.R^{2}=0,392\right)$} \\
\hline Preditor & LIIIC & LS-IC & Estimador & Erro padrão & p-valor \\
\hline Intercepto & $-8,863$ & $-0,123$ & $-3,564$ & 2,369 & 0,050 \\
\hline Experiências prévias & $-0,102$ & 0,243 & 0,054 & 0,089 & 0,485 \\
\hline Convivência com engajados & $-0,154$ & 0,424 & 0,138 & 0,145 & 0,260 \\
\hline Percepção de eficácia & 0,129 & 0,668 & 0,344 & 0,137 & 0,005 \\
\hline Empatia pelos animais & $-0,300$ & 0,206 & $-0,078$ & 0,126 & 0,735 \\
\hline Senso de obrigação moral & 0,278 & 0,831 & 0,635 & 0,144 & 0,000 \\
\hline Percepção de semelhança & $-0,150$ & 0,345 & 0,115 & 0,126 & 0,365 \\
\hline Rec. da capacidade de sofrimento & $-0,692$ & 0,124 & $-0,328$ & 0,218 & 0,185 \\
\hline Rec. da dependência & $-0,263$ & 0,240 & $-0,017$ & 0,125 & 0,865 \\
\hline Reconhecimento do direito & 0,017 & 1,236 & 0,523 & 0,302 & 0,040 \\
\hline \multicolumn{6}{|c|}{ Estimadores dos Dados da Coleta Presencial (Pseudo- $\left.R^{2}=0,455\right)$} \\
\hline Preditor & LI-IC & LS-IC & Estimador & Erro padrão & p-valor \\
\hline Intercepto & $-6,534$ & 1,101 & $-3,526$ & 2,002 & 0,135 \\
\hline Experiências prévias & $-0,177$ & 0,191 & $-0,018$ & 0,093 & 0,845 \\
\hline Convivência com engajados & $-0,216$ & 0,375 & 0,100 & 0,148 & 0,590 \\
\hline Percepção de eficácia & 0,351 & 0,938 & 0,599 & 0,146 & 0,000 \\
\hline Empatia pelos animais & $-0,377$ & 0,405 & $-0,032$ & 0,190 & 0,925 \\
\hline Senso de obrigação moral & 0,170 & 0,782 & 0,528 & 0,159 & 0,005 \\
\hline Percepção de semelhança & $-0,256$ & 0,299 & 0,062 & 0,140 & 0,825 \\
\hline Rec. da capacidade de sofrimento & $-0,335$ & 0,267 & $-0,072$ & 0,151 & 0,750 \\
\hline Rec. da dependência & $-0,148$ & 0,605 & 0,182 & 0,197 & 0,320 \\
\hline Reconhecimento do direito & $-0,630$ & 0,526 & 0,026 & 0,298 & 0,835 \\
\hline
\end{tabular}

Fonte: Dados da pesquisa (2015). 
Tabela 3 Estimadores do modelo Theil-Sen para doação de tempo

\begin{tabular}{|c|c|c|c|c|c|}
\hline \multicolumn{6}{|c|}{ Estimadores do Modelo Global (Pseudo- $\left.R^{2}=0,605\right)$} \\
\hline Preditor & LI-IC & LS-IC & Estimador & Erro padrão & p-valor \\
\hline Intercepto & $-7,986$ & $-2,415$ & $-4,424$ & 1,387 & 0,000 \\
\hline Experiências prévias & 0,009 & 0,236 & 0,100 & 0,060 & 0,035 \\
\hline Convivência com engajados & 0,063 & 0,391 & 0,216 & 0,086 & 0,005 \\
\hline Percepção de eficácia & 0,056 & 0,360 & 0,193 & 0,078 & 0,005 \\
\hline Empatia pelos animais & $-0,207$ & 0,126 & $-0,059$ & 0,085 & 0,615 \\
\hline Senso de obrigação moral & 0,499 & 0,847 & 0,720 & 0,094 & 0,000 \\
\hline Percepção de semelhança & $-0,046$ & 0,259 & 0,100 & 0,083 & 0,245 \\
\hline Rec. da capacidade de sofrimento & $-0,294$ & 0,127 & $-0,091$ & 0,113 & 0,500 \\
\hline Rec. da dependência & $-0,210$ & 0,097 & $-0,064$ & 0,081 & 0,455 \\
\hline Reconhecimento do direito & 0,077 & 0,871 & 0,439 & 0,199 & 0,010 \\
\hline \multicolumn{6}{|c|}{ Estimadores dos Dados da Coleta Online (Pseudo- $\left.R^{2}=0,695\right)$} \\
\hline Preditor & LI-IC & LS-IC & Estimador & Erro padrão & p-valor \\
\hline Intercepto & $-9,367$ & $-0,487$ & $-3,625$ & 2,109 & 0,025 \\
\hline Experiências prévias & 0,009 & 0,277 & 0,127 & 0,066 & 0,035 \\
\hline Convivência com engajados & 0,123 & 0,597 & 0,330 & 0,120 & 0,000 \\
\hline Percepção de eficácia & 0,000 & 0,403 & 0,179 & 0,089 & 0,055 \\
\hline Empatia pelos animais & $-0,230$ & 0,155 & $-0,080$ & 0,103 & 0,590 \\
\hline Senso de obrigação moral & 0,317 & 0,783 & 0,639 & 0,110 & 0,000 \\
\hline Percepção de semelhança & 0,062 & 0,478 & 0,254 & 0,108 & 0,010 \\
\hline Rec. da capacidade de sofrimento & $-0,421$ & 0,344 & $-0,075$ & 0,194 & 0,935 \\
\hline Rec. da dependência & $-0,196$ & 0,185 & 0,000 & 0,096 & 0,920 \\
\hline Reconhecimento do direito & $-0,296$ & 0,683 & 0,195 & 0,240 & 0,410 \\
\hline \multicolumn{6}{|c|}{ Estimadores dos Dados da Coleta Presencial (Pseudo- $\left.R^{2}=0,471\right)$} \\
\hline Preditor & LI-IC & LS-IC & Estimador & Erro padrão & p-valor \\
\hline Intercepto & $-1,078$ & $-1,609$ & $-6,547$ & 2,401 & 0,000 \\
\hline Experiências prévias & $-0,173$ & 0,216 & 0,021 & 0,100 & 0,685 \\
\hline Convivência com engajados & $-0,037$ & 0,575 & 0,184 & 0,159 & 0,115 \\
\hline Percepção de eficácia & $-0,064$ & 0,480 & 0,164 & 0,145 & 0,125 \\
\hline Empatia pelos animais & $-0,319$ & 0,441 & $-0,006$ & 0,201 & 0,910 \\
\hline Senso de obrigação moral & 0,373 & 1,121 & 0,848 & 0,193 & 0,000 \\
\hline Percepção de semelhança & $-0,331$ & 0,220 & $-0,068$ & 0,141 & 0,550 \\
\hline Rec. da capacidade de sofrimento & $-0,679$ & 0,018 & $-0,336$ & 0,180 & 0,070 \\
\hline Rec. da dependência & $-0,479$ & 0,191 & $-0,130$ & 0,177 & 0,460 \\
\hline Reconhecimento do direito & 0,238 & 1,711 & 1,028 & 0,371 & 0,010 \\
\hline
\end{tabular}

Fonte: Dados da pesquisa (2015). 
Na estimação do modelo por contextos, o construto de senso de obrigação moral (online $\beta=0,639$, $\mathrm{p}<0,001$; presencial $\beta=0,848, \mathrm{p}<0,001)$ foi confirmado novamente. Os fatores de interação com grupos sociais envolvidos $(\beta=0,330, p<0,001)$, experiência prévias com animais' $(\beta=0,127, \mathrm{p}<0,05)$ e percepção de semelhança $(\beta=0,254, p=0,01)$ também emergiram como fatores explicativos para o contexto online. A percepção de eficácia $(\beta=0,179, \mathrm{p}=0,055)$ sinaliza influência marginal. No contexto presencial, o reconhecimento do direito animal $(\beta=1,028, p<0,05)$ também emergiu como fator explicativo. O nível de ajuste do modelo por contexto foi considerado satisfatório $(0,695)$ e razoável $(0,471)$, respectivamente. A partir dos testes realizados, discutem-se os resultados, realçando algumas considerações para a gestão das organizações de terceiro setor promotoras da causa animal e a aplicação de suas ações de marketing.

\section{DISCUSSÃO DOS RESULTADOS}

Considerando os resultados alcançados, no geral, percebe-se, primeiramente, que para a doação de dinheiro há uma convergência nos fatores identificados, inclusive de caráter mais racional, enquanto que para doação de tempo há uma maior quantidade e diversificação de fatores individuais mais intrínsecos, principalmente entre os contextos de coleta. Esse resultado converge com a compreensão de que o trabalho voluntário é uma atividade de maior envolvimento e complexidade em sua definição, conforme mencionado por Souza e Medeiros (2012) e com o argumento de Lee e Chang (2007) de que o comportamento de doação de tempo envolve questões mais intrínsecas ao indivíduo, como aspectos emocionais. A doação de dinheiro parece estar relacionada a aspectos mais racionais como de capacidade e direito, por exemplo, e extrínsecos, como variáveis sociodemográficas, verificadas em estudos anteriores mencionados, ou fatores de natureza situacional, como visto no estudo experimental de Martin e Randal (2008). Portanto, o direcionamento de apelos desenvolvidos pela comunicação integrada de marketing dessas organizações precisa ser focado em mensagens racionais ou emocionais de acordo com o comportamento de interesse. Essa sinalização converge com as indicações dos estudos de Orsini, Barboza e Costa (2015).

Verificou-se também que a questão de sociabilidade, inerente aos fatores de experiências prévias e convivência com grupos sociais específicos, foi mais perceptível no âmbito de coleta online, o que é coerente com o fato de que essas organizações possuem uma presença digital forte, muitas vezes, mais expressiva que presencialmente, dada as restrições de recursos e os baixos custos para atuação online. Nesse sentido, é possível que as atividades de marketing dessas organizações em redes sociais virtuais alcancem esse papel de influência social como oportunidade, principalmente em relação ao trabalho voluntário, dado que é nesse âmbito que as organizações conseguem expor sua atuação e alcançar um posicionamento quanto ao trabalho que realizam. Essas verificações por comportamento e por contexto, evidenciando suas particularidades de fatores de influência, são consoantes com as afirmações de Andreasen e Kotler (2008) e Kotler (1994) a respeito da consideração de públicos específicos.

Vale salientar que o fator de senso de obrigação moral em relação aos animais foi revelado como o mais consistente, já que emergiu em todos os contextos e para os dois comportamentos considerados, sinalizando sua relevância para explicar a simpatia e envolvimento com a causa animal. Oportunamente, as organizações, somando esforços às outras esferas da sociedade civil, podem desempenhar um papel desafiador e importante para disseminar essa compreensão, por meio de ações de marketing social, vislumbrando a consolidação desse paradigma moral.

Em nível de discussão mais específico, as duas primeiras hipóteses de pesquisa lançadas (H1 e $\mathrm{H} 2$ ) foram parcialmente confirmadas. Embora haja indicações na literatura internacional de que socialização na infância, experiências positivas de vida relacionadas à causa e maior capital humano são fatores de predisposição à doação, as hipóteses foram totalmente rejeitada para doação de dinheiro à causa animal. Já para doação de tempo, houve confirmação parcial (global e contexto online). Essas constatações reforçam o entendimento de que a diferença existente entre os contextos é oriunda do tipo de resposta obtida (espontânea ou não), o que implicaria na presença de respondentes online mais 
'sensíveis à causa' ou 'predispostos a colaborar'. No geral, os resultados convergem com a literatura que aponta essas relações (e.g. BLOUIN, 2012; MARTIN; RANDAL, 2008; ZAHN-WAXLER; HOLLENBECK; RADKE-YARROW, 1985; WIEPKING; MAAS, 2009).

A hipótese $\mathrm{H} 3$ foi totalmente aceita para o comportamento de doação de dinheiro, conforme pesquisas anteriores de Cheung e Chan (2000) e Bekkers e Wiepking (2011), e aceita globalmente para a doação de tempo, resultado convergente com as indicações dos estudos de Slyke e Brooks (2005) e Bekkers (2010). Considera-se então que a hipótese foi aceita, indicando a necessidade das organizações voltadas à causa animal direcionar seu esforço comunicativo para argumentos centrados na percepção de que cada ação individual de doação possui grande eficácia para atingir o objetivo da organização e o resultado social almejado, de modo que promove grandes impactos positivos para esses beneficiários (os animais), sem requerer contrapartida de grandes esforços. Além disso, é importante que as estratégias de marketing da organização incentivem essa percepção de diferencial em conjunto com a definição de alternativas eficazes de captação de recursos, disponibilizando canais que consigam dar sequência à prospecção realizada no esforço de comunicação.

A quarta hipótese ( $\mathrm{H} 4)$ foi plenamente rejeitada, o que diverge dos resultados dos estudos de Lee e Chang (2007), Wiepking e Maas (2009) e Bekkers (2010) sobre a influência positiva da empatia para doação. A não evidenciação desse fator para doação de tempo, particularmente, surpreendeu. Neste caso, sinaliza-se a necessidade de uma análise mais profunda do construto, inclusive em termos de mensuração, verificando perfis e intensidade de participação e colaboração com a causa animal. Também se sugere a investigação desse fator para a evitação de comportamentos negativos (abandono, maus-tratos etc.), seguindo a ideia de Singer (2014) sobre os deveres positivos (promover ajuda) e negativos (não causar danos) da sociedade em relação aos animais, já que as medidas descritivas do construto foram altas. Esse resultado pode corroborar com a indicação de Orsini, Barboza e Costa (2015) de que apelos negativos de dó e sofrimento animal podem não ser amplamente efetivos. Sendo assim, revela a importância da estratégia de marketing das organizações considerar a questão de segmentação de público, apesar de vislumbrar o alcance de toda a sociedade, definindo critérios de segmentação mais adequados à sua atuação, para além de aspectos sociodemográficos.

Conforme exposto anteriormente, a hipótese H5 foi totalmente aceita para os dois comportamentos analisados e converge com as pesquisas de Skyle e Brooks (2005). O senso de obrigação moral se refere à importância e sentimento de dever particular em adotar comportamentos altruístas, neste caso, em relação aos animais. As discussões sobre consideração moral dos animais, então, parecem importantes, de modo que propagá-las até o alcance da sociedade em geral, poderia promover maior engajamento social pela causa. Reitera-se que para esforços de comunicação mais amplos, apelos relacionados à questão moral podem, então, ser mais efetivos em obter contrapartidas de interesse.

A sexta hipótese da pesquisa (H6) foi totalmente rejeitada para doação de dinheiro, mas emergiu para doação de tempo no contexto online. Sendo assim, pode-se dizer que há uma aceitação parcial, mas limitada da relação prevista. Embora a literatura sinalize que a percepção de semelhança entre doador e beneficiário aumenta as chances de doação, o presente estudo não evidenciou tal fator como determinante para esses comportamentos, assim como o estudo de Rothgerber e Mican (2014), que não evidenciou esse fator como determinante para evitação do consumo de carne, outro comportamento pró-animal. Deste modo, a não constatação desse aspecto mais emocional, assim como o de empatia, reforça a possibilidade de efetividade relativa de mensagens com conteúdo que apela para sofrimento e dó. É possível que conteúdos dessa natureza tornem a causa menos convidativa, seja por desencadear sentimentos negativos, como culpa ou impotência, seja por estimular um 'estado de negação' para neutralizar os possíveis efeitos dos conteúdos gerados pelas organizações. Esse é um aspecto relevante para novos desdobramentos de pesquisa.

A sétima hipótese ( $\mathrm{H} 7)$ foi totalmente rejeitada. O resultado surpreende, mas sinaliza para a possibilidade de investigar relações cruzadas com outras variáveis, como já ter contribuído anteriormente para a causa ou já possuir animais. Além disso, evidencia a possibilidade de que o fator seja 
determinante para comportamentos relacionados a deveres negativos (SINGER, 2014), já que as medidas descritivas também foram elevadas. Vale salientar que tal constatação reforça também a discussão realizada para os construtos de empatia e percepção de semelhança e traz à tona a possibilidade de discussão sobre a fadiga da compaixão, fenômeno que tem sido densamente explorado sob a perspectiva médica, mas pouco discutido como diminuição do desejo de adotar comportamentos de ajuda (DRUCKER, 2006; LINK et al., 1995).

A oitava hipótese lançada (H8) também foi rejeitada totalmente. O reconhecimento da dependência animal envolvia a aceitação de que os animais domésticos dependem das pessoas para garantir sua sobrevivência e promover seu bem-estar. A não confirmação da relação prevista sugere que essa premissa já repercute em alguma medida na sociedade, considerando as medidas descritivas extraídas, mas, não é fator motivador suficiente para obter colaboração efetiva. Neste caso, se faz oportuno explorá-lo também no contexto de evitação de comportamentos negativos ou como antecedente de outros construtos relacionados, como por exemplo, empatia e atitude em relação aos animais.

A última hipótese formulada (H9) foi confirmada no contexto online para doação de dinheiro, e para doação de tempo, foi aceita globalmente e no contexto presencial. Neste sentido, em geral a hipótese foi aceita. Na pesquisa de Orsini, Barboza e Costa (2015) o apelo ao direito animal também sinalizou eficiência para colaboração com causa. Ao que parece essa variável tem influência limitada e por vezes marginal, mas, assim como o construto anterior, repercute em alguma medida na sociedade, considerando as medidas descritivas obtidas, de modo que também pode ser mais representativa para a evitação de comportamentos negativos. É importante ressaltar que novos esforços precisam ser desenvolvidos para a construção de escalas que possam dar maior suporte à verificação desse e de outros construtos estudados, tendo em vista que este estudo configura uma primeira iniciativa e possui limitações nesse aspecto. A seguir, realizamos as considerações finais do estudo, tangenciando esse e outros aspectos.

\section{CONSIDERAÇÕES FINAIS}

O objetivo dessa pesquisa foi analisar fatores individuais que levam à adoção de comportamentos de doação de dinheiro e tempo para a causa animal, considerando a relevância dessa compreensão para o desempenho profissional da gestão de marketing das organizações sociais atuantes nesse contexto. Em geral, constatou-se que as pessoas são mais propensas a contribuir com a causa animal por meio de doação financeira quando percebem que a própria colaboração e a ação de organizações relacionadas à causa são capazes de proporcionar bem-estar aos animais e quando existe um sentimento de importância moral e dever para com os animais, reconhecendo seus direitos legítimos à vida digna e saudável. Em paralelo, há maior propensão à colaboração com atividade voluntária quando, além dos aspectos mencionados, há percepção positiva de experiências de vida relacionadas aos animais, convivência com pessoas engajadas na causa e, em menor grau, identificação de semelhanças entre pessoas e animais domésticos.

A sinalização de que o senso de obrigação moral é fator determinante para doação implica na necessidade de abordá-lo de forma mais representativa no discurso e ações das ONGs voltadas à causa animal. A proposta é de reforçar não somente o papel do poder público e das organizações sociais, mas principalmente de cada agente cidadão, como provedor de bem-estar aos animais e à sociedade de maneira geral.

Conforme exposto anteriormente, o debate acadêmico sobre essa temática é importante à medida que colabora para preencher lacunas referentes ao reconhecimento da aplicação de marketing em outros contextos, para a utilização de conteúdos acadêmicos na prática de gestão organizacional e para a compreensão mais específica de cada contexto de atuação, entre as diferentes finalidades e causas sociais. Nesse sentido, o estudo permitiu compreender, em alguma medida, o que pode levar as pessoas a adotar comportamentos de colaboração com a causa animal, trazendo implicações para a reflexão de estratégias de marketing alinhadas ao público e ao tipo de comportamento desejado, no intuito de gerar maior eficácia no desenvolvimento de suas ações e facilitar o gerenciamento de áreas fundamentais, como gestão financeira e recursos humanos. Por 
exemplo, sabendo que apelos de sofrimento não são tão efetivos quanto se imagina (e esses apelos são muito utilizados pelas entidades de proteção animal), é possível evitar desperdício de esforços e direcioná-los a iniciativas mais atrativas para captação de recursos e estimulantes para adoção de comportamentos sociais desejáveis. Ademais, essa diferenciação captada entre contextos de coleta, também pode evidenciar os perfis de públicos atuantes, de modo que possibilita uma diretriz em termos de atuação online e off-line para essas entidades.

Adotar uma perspectiva de gestão estratégica de marketing possibilita às ONGs perceber na sociedade seus públicos provedores e consumidores, de modo que direcione ações para promover o envolvimento com a causa em detrimento da omissão, partindo da premissa de que não existe uma maioria que maltrata e abusa os animais, mas que é indiferente à causa, conforme sinalizam os resultados. Na esfera acadêmica, o estudo pode servir de referência para elaborações de modelos teóricos menos genéricos e levanta a possibilidade de discussão de outros construtos, pouco ou ainda não explorados em pesquisas da área.

Em relação às limitações do estudo, considerando seu escopo quantitativo, além do que já foi indicado, observa-se uma limitação no tamanho, lócus de pesquisa e perfil da amostra. Apesar de significativa para o propósito exploratório do estudo, resultados mais consistentes poderiam ser alcançados com a captação de mais respostas, principalmente porque se constatou diferença entre contextos e suas respectivas análises foram feitas com número menor de respondentes. Além disso, a aplicação de questionários nos dois contextos de coleta contribuiu para a definição do perfil de amostra com maiores níveis de renda e escolaridade. Deste modo, sugerimos a realização de novos estudos com amostras mais heterogêneas e com quantitativo de respondentes ainda maiores, em nível nacional, explorando inclusive a relação de variáveis sociodemográficas com os comportamentos de interesse.

Salienta-se também a necessidade de novos estudos com melhorias na mensuração dos construtos utilizados, já que alguns nunca foram explorados na literatura em abordagem quantitativa (como percepção de semelhança, reconhecimento da dependência animal etc.) e outros apresentaram medidas que sinalizam essa necessidade, como reconhecimento do direito animal. Isso pode ter prejudicado a constatação de relações mais consistentes.

Outra limitação a ser ressaltada é que o estudo explorou essencialmente a relação linear entre os fatores antecedentes e de resposta. O uso de outras modelagens poderia evidenciar resultados mais precisos, já que houve a rejeição de algumas hipóteses e há sinalizações na literatura de relação entre os próprios fatores antecedentes determinados. Por exemplo, alguns autores (BLOUIN, 2012; ZAHN-WAXLER; HOLLENBECK; RADKE-YARROW, 1985) sugerem que experiências prévias com animais como fator antecedente à empatia por animais.

Ademais, considera-se oportuno o desenvolvimento de estudos com abordagem qualitativa, já que essa se aplica adequadamente a objetos de estudo ainda pouco discutidos, podendo contribuir para o aprofundamento do tema, como por exemplo, investigando essas relações considerando os cenários de iniciação, continuidade ou descontinuidade dos comportamentos de doação para a causa.

\section{REFERÊNCIAS}

AGÊNCIA DE NOTÍCIAS DE DIREITOS ANIMAIS (ANDA). Cresce para 30 milhões o número de animais abandonados no Brasil. 2014. Disponível em: <http://www.anda.jor.br/01/03/2014/cresce-30-milhoes-numero-animais-abandonados-brasil> Acesso em: 10 jan. 2016.

ANDREASEN, A. R.; KOTLER, P. Strategic Marketing for Nonprofit Organizations. 7th ed. New Jersey: Pearson Prentice Hall, 2008.

ASSOCIAÇÃO BRASILEIRA DA INDÚSTRIA DE PRODUTOS PARA ANIMAIS DE ESTIMAÇÃO (ABINPET). 2013. Indústria nacional fatura $\mathbf{R} \$$ 15,2 bilhões e já representa 0,31\% do PIB nacional. Disponível em: <http://abinpet.org.br/imprensa/ noticias/abinpet-divulgadados-mercado-pet-2013> Acesso em: 10 jan. 2016. 
BASIL, D. Z.; RIDGWAY, N. M.; BASIL, M. D. Guilt and giving: a process model of empathy and efficacy. Psychology \& Marketing, v. 25, n. 1, p. 1-23, 2008.

BEKKERS, R. Who gives what and when? A scenario study of intentions to give time and money. Social Science Research, v. 39, n. 3, p. 369-381, maio 2010.

BEKKERS, R.; WIEPKING, P. A literature review of empirical studies of philanthropy: eight mechanisms that drive charitable giving. Nonprofit and Voluntary Sector Quarterly, v. 40, n. 5, p. 924-973, 2011.

BELDAD, A.; SNIP, B.; VAN HOOF, J. Generosity the second time around: Determinants of individuals' repeat donation intention. Nonprofit and Voluntary Sector Quarterly, v. 43, n. 1, p. 144-163, 2014.

BLERY, E. K.; KATSELI, E.; TSARA, N. Marketing for a non-profit organization. International Review on Public and Nonprofit Marketing, v. 7, n. 1, p. 57-68, 2010.

BLOUIN, D. D. Understanding relations between people and their pets. Sociology Compass, v. 6, n. 11, p. 856-869, nov. 2012.

BRANCO, A. U. de A. Comportamento pró-social: análise conceitual e variáveis correlatas. Arquivos Brasileiros de Psicologia, Rio de Janeiro, v. 35, n. 2, p. 153-169, 1983.

BRASIL. Lei $\mathrm{n}^{\circ}$ 9.605, de 12 de fevereiro de 1998b. Lei de Crimes Ambientais. Diário Oficial da União, Brasília, DF, 13 fev. 1988b. Disponível em: < https:// www.planalto.gov.br/ ccivil_03/leis/19605.htm>. Acesso em: 04 abr. 2016.

BRYANT, W. K. et al. Participation in philanthropic activities: Donating money and time. Journal of Consumer Policy, v. 26, n. 1, p. 43-73, 2003.
BURGUETE, J. L. V. Pasado, presente y futuro de las dimensiones pública y social en el desarrollo conceptual del marketing. Revista Internacional de Marketing Público y no Lucrativo, v. 1, n. 1, p. 9-34, jun. 2004.

ČAČIJA, L. N. Fundraising in the context of nonprofit strategic marketing: Toward a conceptual model. Management: journal of contemporary management issues, v. 18, n. 1, p. 59-78, 2013.

CHEUNG, C. K.; CHAN, C. M. Social-cognitive factors of donating money to charity, with special attention to an international relief organization. Evaluation and Program Planning, v. 23, n. 2, p. 241-253, 2000.

COSTA, F. J. da. Mensuração e desenvolvimento de escalas: aplicações em administração. Rio de Janeiro: Ciência Moderna, 2011.

DOLNICAR, S.; LAZAREVSKI, K. Marketing in non-profit organizations: an international perspective. International Marketing Review, v. 26, n. 3, p. 275-291, 2009.

DRUCKER, P. F. Administração de organizações sem fins lucrativos: princípios e Práticas. 1 ed. São Paulo: Pioneira, 2006.

EPLEY, N.; WAYTZ, A.; CACIOPPO, J. T. On seeing human: a three-factor theory of anthropomorphism. Psychological Review, v. 114, n. 4, p. 864-886, out. 2007.

GUY, B. S; PATTON W. E. The marketing of altruistic causes: understanding why people help. Journal of Services Marketing, v. 2, n. 1, p. 5-16, 1989.

HAIR JR, J. F. et al. Fundamentos de Pesquisa de Marketing. São Paulo: Bookman, 2014.

HAYNES, M.; THORNTON, J.; JONES, S. C. An exploratory study on the effect of positive (warmth appeal) and negative (guilt appeal) print imagery on donation behaviour in animal welfare. Research Online, Nova Zelândia, p.1-10, 2004. 
HOLBROOK, M. B. Pets and people: companions in commerce? Journal of Business Research, v. 61, n. 5, p. 546-552, 2008.

INSTITUTO BRASILEIRO DO MEIO AMBIENTE E DOS RECURSOS NATURAIS RENOVÁVEIS (IBAMA). Portaria no 93, de 07 de julho de 1998. Seção 1, p. 74-77. Disponível em: <http://www. redeprofauna.pr.gov.br/arquivos/File/legislacao/ port_93_98.pdf $>$. Acesso em: 20 mar. 2016

INSTITUTO BRASILEIRO DE GEOGRAFIA E ESTATÍSTICA (IBGE). Pesquisa domiciliar sobre cães e gatos: humanização e padrões de consumo CDHPET / Escola Nacional de Ciências Estatísticas. 20. ed. Rio de Janeiro: IBGE, 2007. 81 p.. Disponível em: <http://biblioteca.ibge.gov.br/visualizacao/ livros/liv39560.pdf>. Acesso em: 04 jun. 2015.

INSTITUTO DE PESQUISA ECONÔMICA APLICADA (IPEA). Mapa das organizações da sociedade civil. 2014. Disponível em: <https://mapaosc.ipea.gov.br>. Acesso em: 10 jun 2015.

KOTLER, P. Marketing para organizações que não visam lucro. São Paulo: Atlas, 1994.

KOTLER, P.; LEVY, S. Broadening the concept of marketing. Journal of marketing, v. 33, p. 10-15, jan. 1969.

KOTTASZ, R. Differences in the donor behavior characteristics of young affluent males and females: Empirical evidence from Britain. Voluntas: International Journal of Voluntary and Nonprofit Organizations, v. 15, n. 2, p. 181-203, 2004.

LEE, Y.; CHANG, C. Who gives what to charity? Characteristics affecting donation behavior. Social Behavior and Personality: an international journal, v. 35, n. 9, p. 1173-1180, 2007.

LINK, B. G. et al. Public knowledge, attitudes, and beliefs about homeless people: evidence for compassion fatigue? American Journal of Community Psychology, v. 23, n. 4, p. 533-555, 1995.
MARTIN, R.; RANDAL, J. How is donation behaviour affected by the donations of others? Journal of Economic Behavior \& Organization, v. 67, n. 1, p. $228-238,2008$.

MEDEIROS, F. L. F. de; GRAU NETO, W. A esquizofrenia moral e o dever fundamental de proteção ao animal não-humano. Revista Brasileira de Direito Animal, Salvador, v. 7, n. 10, p. 275-324, 2012

NEUMANN, S. L. Animal Welfare Volunteers: who are they and why do they do what they do? Anthrozoös, v. 23, n. 4, p.351-364, 2010.

ORSINI, A. C. R.; BARBOZA, S. I. S.; COSTA, F. J da. Motivações Para Adoção de Comportamentos Favoráveis à Causa Animal: Um Estudo Experimental. APGS, v. 7, n. 3, p. 158-167, 2015.

POPE, J. A.; ISELY, E. S.; ASAMOA-TUTU, F. Developing a marketing strategy for nonprofit organizations: an exploratory study. Journal of Nonprofit \& Public Sector marketing, v. 21, n. 2, p. 184-201, 2009.

REGAN, T. The rights of humans and other animals. Ethics \& Behavior, v. 7, n. 2, p. 103-111, 1997.

REGAN, T. Utility and equality: Some neglected problems. Journal of Value Inquiry, v. 17, n. 1, p. 33-52, 1983.

ROSSITER, J. R. ER-SERVCOMPSQUAL: a measure of e-retailing service components quality. Service Science, v. 1, n. 4, p. 212-224, 2009.

ROTHGERBER, H.; MICAN, F. Childhood pet ownership, attachment to pets, and subsequent meat avoidance. The mediating role of empathy toward animals. Appetite, v. 79, p. 11-17, 2014.

SINGER, P. Libertação Animal. São Paulo: WMF Martins Fontes, 2010. 
SINGER, P. Entrevista com Peter Singer [ago. 2014]. Entrevistador: Luciano Cunha. Revista Eco 21, Rio de Janeiro, 213. Disponível em: http://www.eco21. com.br/textos/textos.asp? ID=3360. Acesso em: 04 jan. 2016.

SLYKE, D. M. V.; BROOKS, A. C. Why do people give? New evidence and strategies for nonprofit managers. The American Review of Public Administration, v. 35, n. 3, p. 199-222, 2005.

SOUZA, W. J. de; MEDEIROS, J. P de. Trabalho voluntário: motivos para sua realização. Revista de Ciências da Administração, v. 14, n. 33, 2012.

UNESCO. Declaração Universal dos Direitos dos Animais. Bruxelas, 27 jan. 1978. Disponível em: $<$ http://www.propq.ufscar.br/comissoes-de-etica/ comissao-de-etica-na-experimentacao-animal/ direitos>. Acesso em: 02 abr. 2016.

WIEPKING, P.; MAAS, I. Resources that make you generous: effects of social and human resources on charitable giving. Social Forces, v. 87, n. 4, p. 19731995, 2009.
WILCOX, R. R. Modern statistics for the social and behavioral sciences: a practical introduction. Kentucky: CRC Press, 2012.

WYMER, W.; KNOWLES, P.; GOMES, R. Nonprofit marketing: marketing management for charitable and nongovernmental organizations. California: Sage Publications, 2006.

ZAHN-WAXLER, C.; HOLLENBECK, B.; RADKEYARROW, M.. The origins of empathy and altruism. In: FOX, M. W.; MICKLEY, L. D. (Ed.). Advances in Animal Welfare Sciente 1984. Boston: Martinus Nijhoff Publishers, 1985. p. 21-41.

ZUCCO, F. D.; KRAUSS, I. B.; FERNANDES, V. Apelo e persuasão publicitária no contexto de uma ONG de bem-estar animal: perspectivas para o desenvolvimento de campanhas sociais. Razón y Palabra, v. 18, n. 84, 2013. 


\section{APÊNDICE A - MEDIDAS DESCRITIVAS GLOBAIS DOS FATORES}

\begin{tabular}{|c|c|c|c|c|c|c|c|c|c|}
\hline \multirow{2}{*}{ Construto } & \multicolumn{6}{|c|}{ Medidas de posição } & \multirow{2}{*}{$\begin{array}{l}\text { Medida } \\
\text { de disp. } \\
\text { Desvio } \\
\text { padrão }\end{array}$} & \multicolumn{2}{|c|}{ Medidas de forma } \\
\hline & Méd. & $\begin{array}{l}\text { Méd. } \\
\text { Ap. }\end{array}$ & $\begin{array}{l}\text { Méd. } \\
\text { Wins. }\end{array}$ & Q1 & Q2 & Q3 & & $\begin{array}{l}\text { Coef. de } \\
\text { assimet. }\end{array}$ & $\begin{array}{l}\text { Coef. de } \\
\text { curtose }\end{array}$ \\
\hline \multicolumn{10}{|c|}{ Variáveis preditas } \\
\hline Predisposição à doação de dinheiro & 6.1 & 6.3 & 6.2 & 4,0 & 6.3 & 8.7 & 2.8 & -0.38 & 2.13 \\
\hline Predisposição à doação de tempo & 6.6 & 6.9 & 6.8 & 4.7 & 7.3 & 9.3 & 2.8 & -0.61 & 2.33 \\
\hline \multicolumn{10}{|c|}{ Variáveis preditoras } \\
\hline Exp. prévias com animais & 8,2 & 8,6 & 8,4 & 7,0 & 9,0 & 10,0 & 2,3 & $-1,3$ & 3,9 \\
\hline Int. com grupos sociais & 3,0 & 3,0 & 3,0 & 2,0 & 3,0 & 4,0 & 1,1 & 0,0 & 2,3 \\
\hline Percepção de eficácia & 7,1 & 7,2 & 7,2 & 6,0 & 7,5 & 8,5 & 1,9 & $-0,5$ & 2,7 \\
\hline Empatia por animais & 8,0 & 8,2 & 8,1 & 6,5 & 8,5 & 9,8 & 2,0 & $-0,9$ & 3,1 \\
\hline Senso de obrigação moral & 7,4 & 7,7 & 7,6 & 5,8 & 8,0 & 10,0 & 2,5 & $-0,9$ & 3,0 \\
\hline Percepção de semelhança & 8,5 & 8,9 & 8,7 & 7,8 & 9,0 & 10,0 & 1,8 & $-1,8$ & 6,5 \\
\hline Rec. da cap. de sofrimento animal & 9,2 & 9,5 & 9,4 & 9,0 & 10,0 & 10,0 & 1,4 & $-2,4$ & 8,9 \\
\hline Rec. da dependência animal & 9,0 & 9,3 & 9,1 & 8,5 & 9,5 & 10,0 & 1,3 & $-1,6$ & 5,5 \\
\hline Rec. do direito animal & 9,6 & 9,9 & 9,7 & 10,0 & 10,0 & 10,0 & 1,0 & $-3,7$ & 19,4 \\
\hline
\end{tabular}

